\title{
Stalking a new target
}

Sepsis is a potentially lethal systemic condition that arises from a dysregulated host immune response to infection and for which effective, targeted therapies are lacking. A recent study by Tang and colleagues has identified anaplastic lymphoma kinase (ALK) as an important driver of immune responses during sepsis and has shown that inhibition of this target confers protection in animal models.

During the development of sepsis, the innate immune system becomes activated in response to recognition of microbial motifs, such as cyclic dinucleotides, triggering an inflammatory response that becomes dysregulated. The stimulator of interferon (IFN) genes (STING) a transmembrane adaptor protein

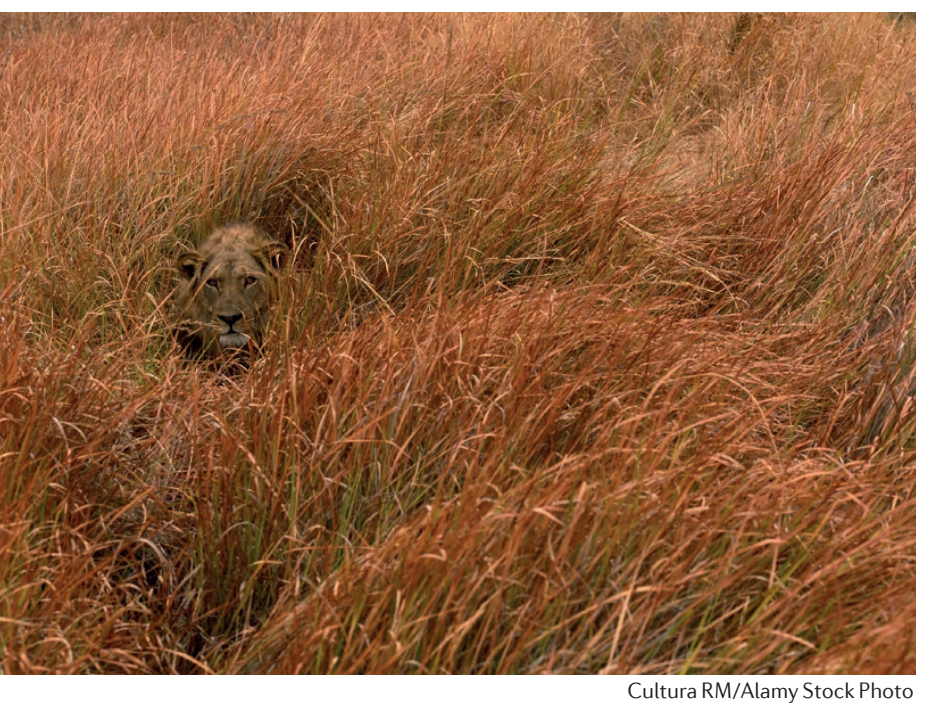

that regulates production of type I IFNs - is a key player in the innate recognition system, and recent evidence in mouse models suggested STING signalling might accelerate the development of sepsis.

Tang and co-workers began their study by screening a library of 464 kinase inhibitors to look for STING-modulating compounds. Compounds were tested in immortalized bone marrow-derived macrophages (iBMDMs) from B6 mice for their ability to alter IFN $\beta$ production in response to a cyclic dinucleotide. Two of the top five compounds that blocked IFN $\beta$ release (AZD3463 and LDK378) were found to be inhibitors of ALK, a tumour-associated receptor tyrosine kinase (RTK). Moreover, among the 174 molecular targets of the compound library, ALK was the top-ranked driver of cyclic dinucleotide-induced STING activation.

Next, the authors showed that LDK378 and another ALK inhibitor, AP26113, inhibited IFN $\beta$ release in iBMDMs and in several cell lines in response to a range of STING ligands. Genetic knockdown of ALK using short hairpin RNA had similar effects.

Results from co-immunoprecipitation studies suggested ALK and STING do not directly interact with one another. Rather, the team found evidence that ALK interacts with epidermal growth factor receptor (EGFR), another cell surface RTK, which promotes STING activation via downstream signalling through AKT. Consistent with these findings, pharmacological inhibition of the ALK-EGFR-AKT pathway reduced STING ligand-induced release of IFN $\beta$ in iBMDMs.

Importantly, these results translated to the in vivo setting. In the cecal ligation and puncture mouse model of polymicrobial sepsis, intraperitoneal injection of LDK378 at 2, 24, 48 and 72 hours protected against lethality and reduced injury in organs including the lung and small intestine. Biochemical measurement of specific tissue enzymes also indicated protection in the heart, pancreas, liver and kidneys. Similarly beneficial effects of LDK378 were seen in a lipopolysaccharide-induced mouse model of endotoxaemia.

Last, Tang and colleagues showed that expression of the ALK-STING pathway is upregulated in peripheral blood mononuclear cells from patients with sepsis, supporting the clinical relevance of their findings.

The current study presents ALK as a novel potential target for sepsis and suggests clinical testing of LDK378 (which is also known as ceritinib and is approved by the FDA for ALK-positive non-small-cell lung cancer) is warranted in this setting.

Katie Kingwell

ORIGINAL ARTICLE Zeng, L. et al. ALK is a therapeutic target for lethal sepsis. Sci. Transl Med. 9, eaan5689 (2017) 